\title{
Diffractive optical elements made from photonic metamaterials
}

Thomas Pertsch, Benny Walther, Stefan Fasold, Matthias Falkner, Christoph Menzel, et al.

Thomas Pertsch, Benny Walther, Stefan Fasold, Matthias Falkner, Christoph Menzel, Isabelle Staude, Carsten Rockstuhl, "Diffractive optical elements made from photonic metamaterials," Proc. SPIE 9626, Optical Systems Design 2015: Optical Design and Engineering VI, 962604 (23 September 2015); doi: 10.1117/12.2195726

SPIE. Event: SPIE Optical Systems Design, 2015, Jena, Germany 


\title{
Diffractive optical elements made from metamaterials
}

\author{
Thomas Pertsch ${ }^{1, *}$, Benny Walther ${ }^{1}$, Stefan Fasold ${ }^{1}$, Matthias Falkner ${ }^{1}$, Christoph Menzel ${ }^{1}$, \\ Isabelle Staude ${ }^{1}$, Carsten Rockstuhl ${ }^{2}$ \\ ${ }^{1}$ Institute of Applied Physics, Abbe Center of Photonics, Friedrich-Schiller-Universität Jena, \\ Max-Wien-Platz 1, 07743 Jena, Germany; \\ ${ }^{2}$ Karlsruhe Institute of Technology, Institute of Theoretical Solid State Physics, \\ Wolfgang-Gaede-Str. 1, 76128 Karlsruhe, Germany; \\ * Corresponding author, email: thomas.pertsch@uni-jena.de, phone: +49 (0)3641 947840
}

\begin{abstract}
The excitation of surface plasmon polaritons in metallic nanostructures significantly enhances light-matter interactions at sub-wavelength scales. This enables novel optical effects that rely on artificial materials, so-called metamaterials. Metamaterials are made from densely packed and sufficiently small nanostructured unit cells. The purpose of metamaterials is to act comparable to bulk materials but with effective properties that can be tailored by the geometry of the unit cells. However, recently it became obvious that many appealing applications are already in reach by employing an ultrathin layer of metamaterial. Exploiting the metamaterials' primary optical properties in the form of their dispersive complex reflection and transmission coefficients, single functional layers instead of bulk metamaterials are already sufficient for achieving sophisticated device properties. In particular, if the unit cells change across the functional layer, a new class of devices can be perceived that shape the light in the far-field according to predefined patterns. These metamaterial layers, usually referred to as metasurfaces, possess major advantages when compared to traditional optical elements. Here, we provide an overview of the burgeoning field of research that explores metasurfaces to affect an incident field spatially and spectrally in a deterministic way to enable functional diffractive optical elements.
\end{abstract}

Keywords: Diffractive optics, holograms, metamaterials, metasurfaces, plasmonics, nanomaterials

\section{INTRODUCTION}

Intense light-matter-interaction using metallic nanostructures is the key to tame light at scales much smaller than the wavelength. Especially metamaterials, understood here as arrays of nanostructures made from metals and with optical properties beyond those of natural materials, have been used to bend, deflect, focus, transmit, or otherwise shape electromagnetic waves. These light-shaping elements became progressively thinner and thinner and can be designed for operating wavelengths from the microwave regime down to the visible. Simultaneously, their functional flexibilities caught up with and exceeded the capabilities of traditional diffractive optical elements. Typical tasks which belonged to the domain of classical dielectric gratings and diffractive elements, such as light bending, lensing, imaging and holography, can now be performed by ultrathin metamaterial layers ${ }^{1,2}$. While on the one hand metamaterials are entering the world of diffractive elements and computer-generated holograms, the already existing know-how and concepts developed in the field of diffractive optics on the other hand is often tapped as a source for the design of new metasurfaces. Thus, as these two fields merge, so do the different science communities. Therefore, this overview is written to address both communities, the metamaterial and the diffractive optics community, and to highlight the recent advances made in metamaterial-based diffractive optical elements. While some of the presented aspects will be wellknown in one community, their inclusion might yet be indispensable for the other.

The starting point of our introduction shall be the appreciation that most metamaterials' properties are derived from surface plasmon polaritons that are sustained by the individual unit cells of the metamaterial. We distinguish between propagating and localized surface plasmon polaritons. While the former are associated to guided modes at extended interfaces between metals and dielectrics, the latter are supported by isolated metallic nanostructures. The excitation of a surface plasmon polariton modifies the properties of electromagnetic fields such as the amplitude, the field gradient, or the local density of states in the close proximity of the metallic structure. This has consequences for quantities accessible in the far-field such as reflection, transmission, scattering, or absorption. How these properties are affected depends

Optical Systems Design 2015: Optical Design and Engineering VI, edited by Laurent Mazuray,

Rolf Wartmann, Andrew P. Wood, Proc. of SPIE Vol. 9626, 962604 - (c) 2015

SPIE · CCC code: $0277-786$ X/15/\$18 $\cdot$ doi: 10.1117/12.2195726 
sensitively on the geometry of the metallic structure as well as on the wavelength and the polarization of the illumination. The response is usually highly dispersive.

The exploitation of surface plasmon polaritons is not an achievement of modern ages, though. The famous Lycurgus cup, made about two-thousand years ago from glass with metallic nanoparticle inclusions, is one of the oldest (unwitting) uses of plasmonics ${ }^{3}$. The cup is perceived with different spectral characteristics depending on the position of the illumination and the observer. A sound scientific explanation for these effects only became possible by the theoretical and experimental works of $\mathrm{Mie}^{4}$, Rayleigh ${ }^{5}$, and Faraday ${ }^{6}$ roughly more than a century ago.

The advent of nanofabrication techniques in the last decades spurred a huge boom in the field of nanoplasmonics. Of particular relevance for our overview of the subject is the opportunity to fabricate metallic nanostructures with complicated shapes, critical dimensions smaller than the optical wavelength, and at sufficiently high densities. This allows to consider the resulting medium as an effective medium with a defined optical response. The intrinsic properties of the used materials, usually metals with a high density of free electrons such as gold, silver, and aluminum, have tremendous effects on the optical response of the metamaterial. However, an equal or even greater effect on its properties has the shape and size of the unit cells of the metamaterial. Proper design allows for tailoring effective electric and magnetic properties independently, resulting in metamaterials with an unprecedented spectral behavior ${ }^{7}$. In particular, unit cells sustaining magnetic and higher-order electric resonances could be synthesized, leading to counterintuitive effects such as negative refraction. Suggested applications of metamaterials comprise super-lenses ${ }^{8}$, cloaking devices ${ }^{9-11}$, zero-reflection media ${ }^{12,13}$, and plasmonic rulers ${ }^{14}$. Also, materials may present truly unconventional properties such as a huge chirality ${ }^{15-18}$ or asymmetric transmission ${ }^{19}$. For most of these applications bulk materials are desirable ${ }^{20,21}$. Unfortunately, fabricating three-dimensional metamaterial brings up two fundamental problems that are challenging to mitigate.

First, the fabrication of bulk metamaterials, where unit cells are not just repeated in a two-dimensional plane but also in a third dimension, remains complicated. While planar metamaterials made from isolated layers can be fabricated by electron beam lithography ${ }^{22}$, direct laser writing techniques ${ }^{23}$ or nanoimprint technology ${ }^{24}$, stacking of individually fabricated functional layers is rather time consuming and expensive. A possible alternative might consist in the exploitation of chemistry-inspired bottom-up methods ${ }^{25,26}$. But this research is in its infancy and it remains an open question whether this route can lead to sufficiently dispersive materials.

The second issue with three-dimensional metamaterials is more on the theoretical side. It concerns the question whether these materials can be considered as truly homogenous and whether effective material properties can be assigned. Effective material properties, such as permittivity and permeability tensors, provide a coherent description of the material independent on the specific illumination or geometry of the device which contains the metamaterial. This requires the electromagnetic response of the metamaterial to be local. This means that the unit cells have to be densely packed and significantly sub-wavelength such that the induced polarization is a local function of the electromagnetic field which does not depend on the field from distant points or on field gradients ${ }^{27}$. Unfortunately, most metamaterials seemingly do not meet this requirement.

However, and this is important in the context of this review, to exploit the unique peculiarities of the nanostructured unit cells in an actual application, a description of the metamaterial in terms of material parameters is not necessary. To make use of metamaterials in an application, it is fully sufficient to consider thin layers of metamaterial as means to independently control reflection and transmission in amplitude and phase. Such monolayers, or metasurfaces, provide a sufficiently strong response and are accessible with state-of-the-art nanofabrication techniques.

Moreover, since nearly all fabrication techniques work in serial, it became possible to not just write a repetition of identical unit cells. Instead, the geometry of the unit cells can be changed smoothly or abruptly across the interface. Consequently, the structure can possess locally varying and tunable optical properties affecting the light's amplitude, phase, polarization as well as the spectral behavior of these quantities in reflection and transmission.

From a conceptual point of view, a generic optical system can be understood as a device that modifies an ingoing wave front to an outgoing wave front. This modification can be done by a medium of virtually no thickness by using metamaterials. This is in stark contrast to ordinary optical elements which usually require the propagation of light through extended bulky materials to let it accumulate a sufficient phase delay and/or to modify its amplitude. Hence, compactness and comprehensive light control are the striking features of metasurfaces.

Here, we provide an overview on recent efforts to achieve metasurfaces that shape light at optical frequencies, often using concepts known from the field of diffractive elements. We explicitly distinguish different streams of research that utilize different physical effects. In a first part we consider metasurfaces made from structures that sustain localized 
plasmon polaritons designed to affect the local phase of the transmitted or reflected light. In a second part we discuss structures that rely on propagating plasmon polaritons in metallic waveguides which likewise aim to affect the phase of the light. Eventually, we present a class of metasurfaces that modulates not just the phase but the amplitude as well. These metasurfaces explicitly take advantage of the absorbing nature of the metallic constituents and turn it into something useful. Even further advanced we also consider structures which control light not just at a single but at multiple frequencies independently. This aspect is discussed with some examples, indicating a promising path towards the full control over light in the spatial and spectral domain. Such metasurfaces might find applications in broad-band pattern generation, as e.g. required for optical data storage, broad-band adaptive optics, multi-dimensional imaging and microscopy, as well as in cryptography and security applications. Therefore, they constitute an essential contribution to the future architecture of many photonic devices.

\section{OPTICAL NANOANTENNA BASED STRUCTURES}

Compared with metals the interaction of light with dielectrics is rather weak. This had been a clear advantage when using dielectric materials to form classical diffractive optical elements such as gratings, Fresnel-lenses, and computergenerated holograms (CGHs), because the marginal absorption in dielectrics is an asset when designing high-efficiency devices. However, for most optical components a phase modulation in the range of $2 \pi$ is required. Until recently achieving these large phase modulations with the limited refractive indices of dielectrics required pronounced surface height modulations ${ }^{28}$. This led to rather thick optical elements and large aspect ratios in the microstructured surfaces. Furthermore, the dispersive properties of dielectrics are intrinsically fixed and usually do not qualify as a free design parameter.

On the other hand, with their vast free charge carrier densities metallic nanostructures interact quite strongly with light due to the excitation of localized plasmon polaritons. The resulting damping is considerable, but can be acceptable for very thin metamaterial surfaces or for off-resonant operation. The phase changes induced by a single layer of metamaterial happen within a few nanometers. In fact, the phase modulation is so abrupt that the metamaterial layer can be considered as an interface between the incident and the transmitted medium representing a phase discontinuity. When the phase modulation is not uniform across the interface but a phase gradient is evoked by geometrical variations in the metamaterial, light can be controlled and deflected. The situation can be described by a reformulation of the classical Snell's law of refraction ${ }^{29}$. With the phase discontinuity at the interface, the relation of incidence angle $\theta_{\mathrm{i}}$ and transmittance angle $\theta_{\mathrm{t}}$ is augmented by an additional term, which is the lateral phase gradient $\mathrm{d} \varphi / \mathrm{d} x$ in the plane of incidence at the particular point of the interface. Therefore, the generalized Snell's law reads as

$$
n_{\mathrm{t}} \sin \left(\theta_{t}\right)=n_{\mathrm{i}} \sin \left(\theta_{i}\right)+\frac{\lambda_{0}}{2 \pi} \frac{\mathrm{d} \phi}{\mathrm{d} x},
$$

where $\lambda_{0}$ is the vacuum wavelength. This behavior has been coined 'anomalous refraction' and a similar equation holds for reflection ${ }^{29}$. Apparently, even at normal incidence a deflection of the transmitted beam is possible. This behavior is in analogy to a blazed grating which redirects light asymmetrically into the $-1^{\text {st }}$ and $+1^{\text {st }}$ diffraction order. However, here the deflection is realized practically by a surface rather than by propagation effects through an extended medium. Equation (1) therefore contains the recipe to design various compact optical devices by realizing corresponding phase gradients. Later it was shown that the anomalous refraction and reflection phenomenon can produce even beams which are out-of-plane with respect to the incidence plane ${ }^{30}$.

The local transmission function, and thus the phase function, of the metasurface is adjusted by choosing properly shaped nanostructures. These structures can be understood as plasmonic antennas or resonators ${ }^{31-33}$. Besides an electric dipolar resonance, as it would be the case for a metallic nanostructure in the quasi-static limit, the antennas in the unit cells often sustain more than a single resonance which may be characterized by magnetic dipole or higher order electric multipole moments $^{34}$. The required phase changes across the surface can conveniently be achieved at wavelengths near the resonance of an optical resonator. There, the phase shift between incident and transmitted wave is very sensitive to the geometry of the resonator. This is because the resonance marks the delimitation between a range of out-of-phase oscillation of incident and scattered field at long wavelengths, and in-phase oscillation at short wavelengths. Thus, the transition region is accompanied by a rapid phase change of around $\pi$. Hence, adjusting the resonance position by tuning the geometry allows to shift the steep phase gradient, resulting in a strong variation of phase at a particular wavelength. 
Often as a side effect of creating geometrically elaborated shapes and breaking of symmetries the nanostructures become anisotropic - incoming linearly polarized light is turned into elliptically polarized light. This can be seen as both, blessing and curse. The blessing clearly is that flexibility and more variety is gained in the optical response of a selected polarization ${ }^{35,36}$. In Ref. $29 \mathrm{~V}$-shaped optical antennas with variable opening angles and arm lengths were used to induce a phase change in the cross-polarized transmitted light when illuminated with linearly polarized light, see Figure 1 . The V-antennas support two orthogonal, independent current modes, symmetric and antisymmetric, in their two arms ${ }^{37}$. By properly choosing the values of opening angle, arm lengths, and orientation it was possible to obtain a set of metamaterial unit cells which induce phase shifts from 0 to $2 \pi$ with a spacing of $\pi / 4$. Meanwhile, the amplitude effect was designed to be roughly the same for each type of antenna. By arranging the different nanoantennas across a plane, a unidirectional phase gradient was established, Figure $1 \mathrm{~b}$. This provides the function of a light-deflecting prism or blazed grating at which the anomalous refractive behavior could be demonstrated, Figure 1c. The curse of anisotropic metasurfaces is, however, that the illuminating wave is partly transmitted as well and needs to be filtered out after the device. This brings about a loss in energy and, in a way, counteracts the advantage of the metasurfaces' compactness. To be specific, for an operational wavelength of $8 \mu \mathrm{m}$ approximately $10 \%$ of the incident radiation experienced the anomalous refraction in transmission and 20\% experienced anomalous reflection in the cross-polarization, $20 \%$ and $40 \%$ remained co-polarized with respect to the illumination and experienced ordinary refraction and reflection respectively, and $10 \%$ antenna absorption has been encountered ${ }^{38}$. For future applications where compactness is essential, integrating the polarizer into the metasurface, e.g. as a second layer, might remedy the problem.
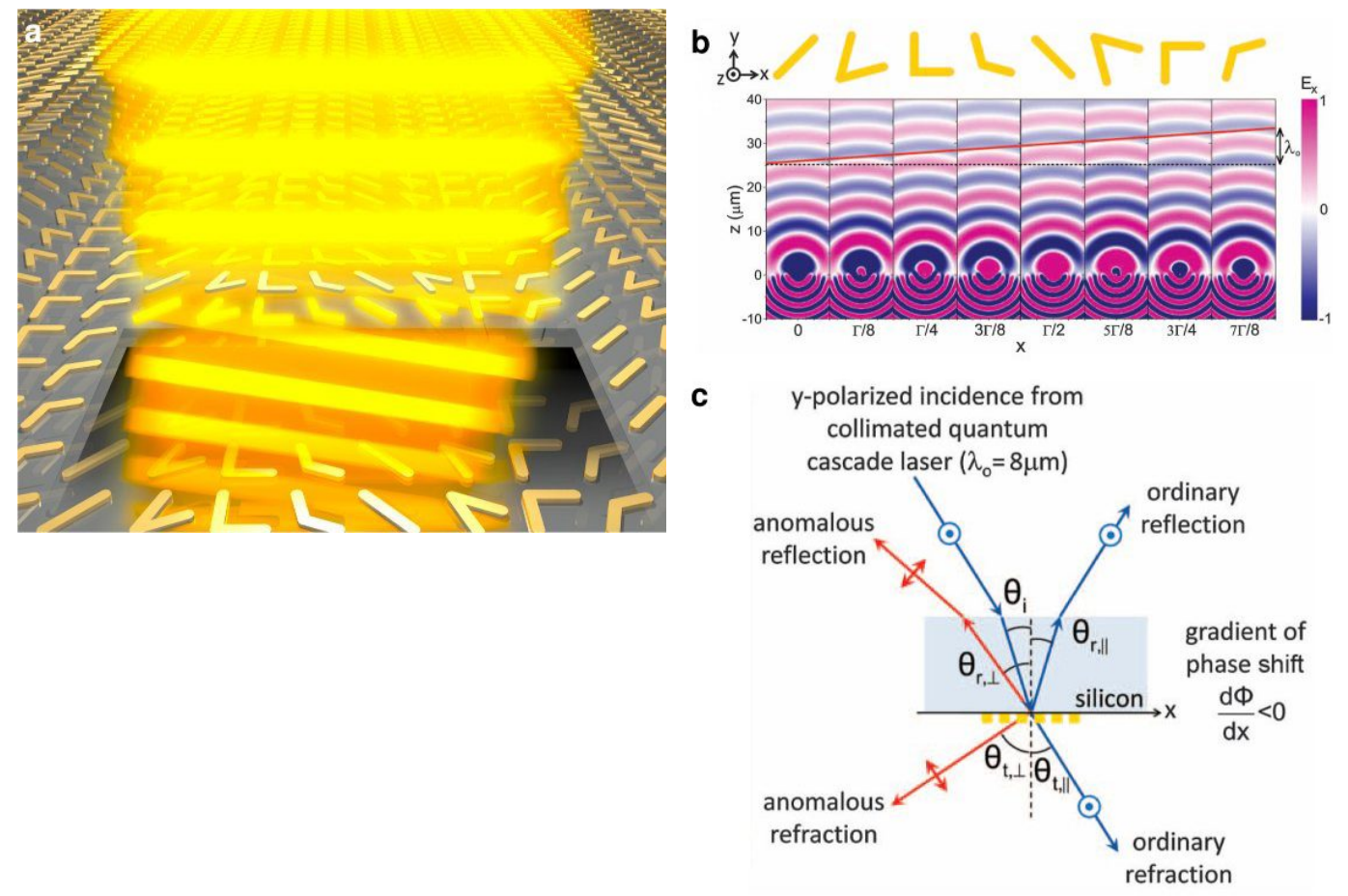

Figure 1. (a) A metasurface with variable V-shaped nanoantennas can deflect light in an abrupt manner by inducing a phase gradient along the surface. In passing we note that the artistic image is slightly idealistic. While properly illustrating the anomalous refraction phenomenon, on purpose we neglected proper representation of polarization and other transmitted or reflected waves to capture the essential of the functionality. (b) By employing eight different antenna version in a unit cell of size $\Gamma$, a phase gradient of $2 \pi / \Gamma$ is approximated. The nanoantennas differ in arm length, opening angle, and orientation. Illumination with y-polarized light produces a phase wedge in the transmitted x-polarized light. (c) For general incident angles ordinarily and anomalously refracted and reflected beams occur, respectively. Figures (b) and (c) reproduced from Ref. 29, (c) 2011 AAAS.

Nevertheless, the idea carries potential for a great many applications. For example, the same set of V-antennas could be arranged in sections of a pie to create an ultra-thin vortex phase mask ${ }^{29,39}$ as shown in Figures $2 \mathrm{a}$ and $\mathrm{b}$. By further shrinking the size of the antennas, the operation wavelength range of 5 to $10 \mu \mathrm{m}$ could be scaled down to the range of 1.0 
to $1.9 \mu \mathrm{m}^{40}$. These antennas can be organized to create nearly arbitrary phase functions, such as that of lenses or axicons $^{41}$. It has to be stressed, though, that the efficiencies of those devices are not yet comparable to those of ordinary dielectric-only devices. The price for the achieved flatness is paid for with additional dissipation in the metallic constituents. For example, the efficiency of the lens as reported in Ref. 41 is only in the order of a percent ${ }^{42}$. Note that energetic efficiency is often not the main focus of these proof-of-principle studies and performance numbers are sometimes difficult to compare. However, an increase of the efficiency towards ten percent or even higher values for this specific lens, and similarly for other devices, is theoretically feasible by shrinking antenna spacing and so the packaging density. This allows to encounter a stronger dispersion which potentially allows to operate the device spectrally far away from the resonance where absorption is less severe. Alternatively, different plasmonic materials are currently in the focus of interest where absorption is reduced, albeit at slightly longer operational wavelengths than those accessible with metals $^{43,44}$.
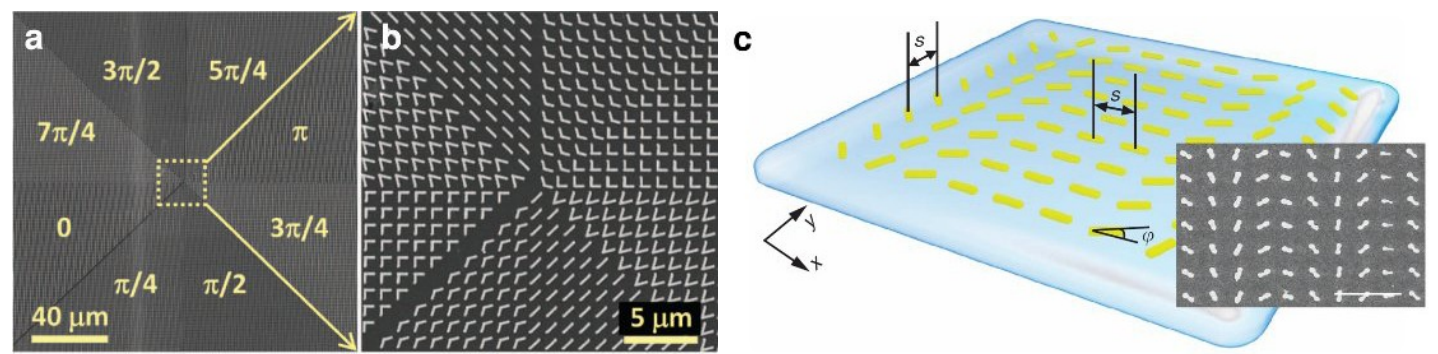

Figure 2. (a) and (b) V-shape nanoantenna elements arranged as sections of a pie to form a vortex mask with discrete phase levels. (c) Linear dipole nanoantennas with varying orientation form a lensing element. For illumination with RCP incident light the metasurface acts as a focusing lens for the LCP transmitted light, while for LCP incident light the metasurface acts as a diverging lens for the RCP transmitted light. The scale bar in the scanning electron microscopy image is $1 \mu \mathrm{m}$. Figures (a) and (b) reproduced from Ref. 29, (C) 2011 AAAS. Figure (c) adapted from Ref. 46, (C) 2012 NPG

Light modulation with such antenna structures is not restricted to phase manipulation, though. Keeping control over the phase influence as adjusted and exerted by the two oscillation modes in the arms, an additional degree of freedom was gained by adding a tail to the $\mathrm{V}$-antenna, making it a $\mathrm{Y}$-shape antenna, which allows to purposefully modulate the amplitude as well ${ }^{45}$.

However, complicated antenna shapes, which often go along with a more pronounced sensitivity to fabrication tolerances, are not indispensable. Abandoning the linear polarization basis and going to circularly polarized (CP) light, a simple electrical dipole antenna can be used to change the phase of transmitted light. The electric dipole moment of a single dipole, forming an angle $\varphi$ with the x-axis, at normally incident CP light is

$$
\boldsymbol{P}_{L(R)}=\frac{1}{\sqrt{2}} \alpha\left(\boldsymbol{e}_{L(R)} \pm e^{ \pm i 2 \varphi} \boldsymbol{e}_{R(L)}\right)
$$

where $\boldsymbol{e}_{L}$ is the unit vector for left-handed circularly-polarized (LCP) incident light, $\boldsymbol{e}_{R}$ for right-handed circularlypolarized (RCP) light, and $\alpha$ is the electric polarizability of the dipole. Hence, for RCP incident light the excited lefthanded polarization experiences a phase delay given by the orientation $\varphi$ of the dipole. With access to the full $2 \pi$ phase range this allows for constructing lenses with an additional, peculiar feature. Straight dipole nanoantennas of fixed length could be arranged to form a lens which is convex for RCP light and concave for LCP light ${ }^{46,47}$. Each dipole nanoantenna had an position-dependent in-plane rotation angle as shown in Figure 2c for the one-dimensional ('cylindrical') lens version. The lens transforms incident RCP light into focused LCP light while, according to Equation (2), for incoming LCP light RCP polarization with the other sign in the phase exponent is created, leading to a divergent light bending. With an operation wavelength of $740 \mathrm{~nm}$ the lens is already close to visible light applications. The practical efficiency of these devices is at around 5 percent ${ }^{47}$.

The shape of the antennas is not limited to straight rods, V- or Y-shape. In a numerical study the electric and magnetic resonances supported by split-ring aperture antennas were exploited ${ }^{48}$. Choosing a wavelength where both resonances appear equally strong in their effect on the transmission amplitude and using the in-plane rotation of the split ring apertures to adjust the phase effect on CP light, nearly full polarization conversion was achieved (RCP to LCP or vice versa). By arranging an appropriate phase gradient, anomalous light deflection or focusing could be realized. 
For many applications the change of polarization is not desired, though. With considerations from radio-frequency, lightbending and focusing metasurfaces could be constructed by means of compound, cubic building blocks without affecting the transmitted polarization at the price of an increased device thickness ${ }^{49}$.

Nevertheless, beyond the application of these mentioned nanoantennas, a large variety of other elements can be safely estimated to be of comparable use. The ability to strongly scatter the light upon excitation of a localized plasmon polariton is the unifying aspect that links the different implementations.

\section{PLASMONIC WAVEGUIDE BASED STRUCTURES}

Then again there are light-shaping elements that are based on propagating surface plasmons or channel-waveguide plasmons. Here the light manipulation is accomplished during propagation through a nanostructured surface of nonnegligible thickness. The concept of adjusting the phase delay with such optical elements has been adopted from the classical efforts with dielectrics. There, height profiles usually made of silica and other dielectrics $\left(\mathrm{TiO}_{2}, \mathrm{PMMA}\right)$, etched with e-beam lithography (EBL) or direct-write technologies, were used to spatially vary the phase delay across the sample, e.g. in order to create lenses or (blazed) gratings. However, the phase modulation is imposed by relying on rather pronounced surface modulations. This is especially true in the case of high efficiency gratings and challenges certain aspects in the practicability of the thin-element approximation ${ }^{50}$. Since fabricating multiple height levels requires several fabrication steps or grey-scale EBL, techniques exploiting effective media based on binary structured dielectrics have been explored ${ }^{51-56}$. The required unit cell substructures are sub-wavelength in the lateral directions, i.e. forming pillars or holes with high aspect ratios as presented in Figures $3 a$ and $b$. The widths of the pillars control the effective content of dielectric seen by the light wave, and thus the phase delay experienced by the wave.
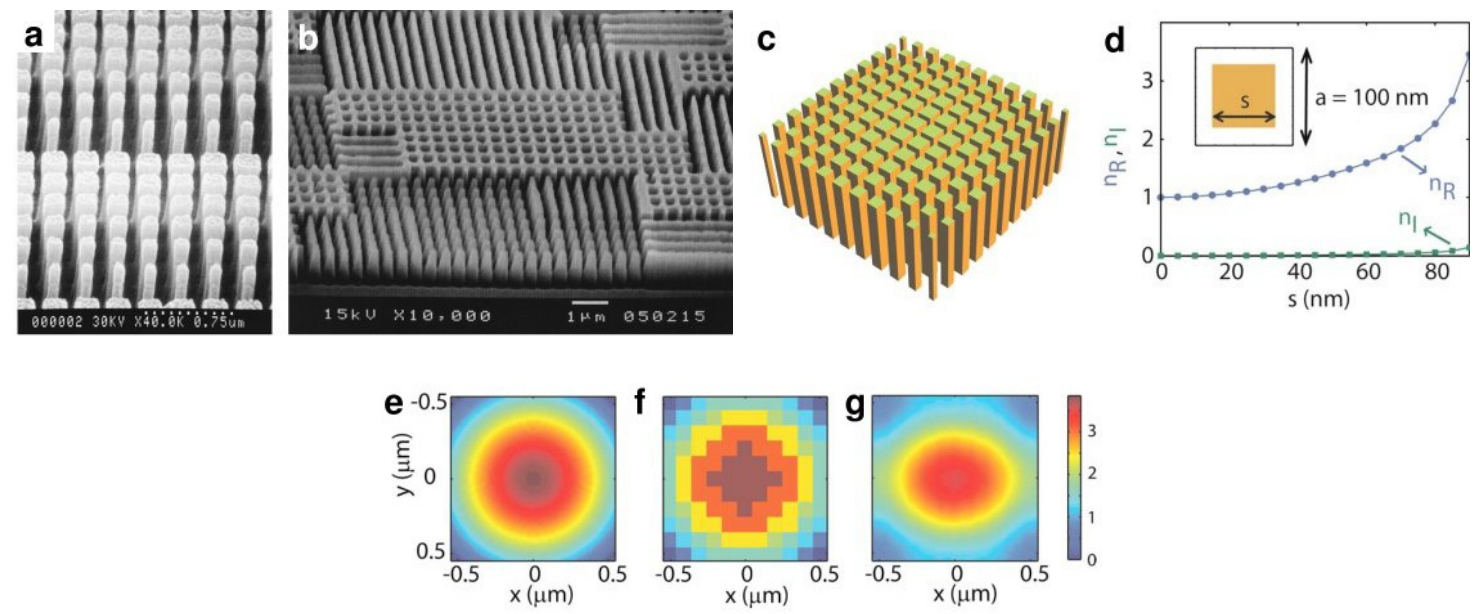

Figure 3. (a) Blazed grating fabricated with binary lithography. The effective index is tuned by the lateral dimensions of the $\mathrm{TiO}_{2}$ pillars. (b) Holographic element with birefringent, subwavelength structures written in fused silica. (c) Microlens design based on metallic pillars. (d) The propagation constant of the light guided in the channels between the pillars, in terms of the effective refractive index $n=n_{R}+i n_{I}$, is strongly affected by the lateral size $s$ of the pillars. With this approach the ideal phase profile (e) of the lens can be approximated by a discretized version (f). The simulated phase front of the device is shown in (g). Figure (a) reproduced from Ref. 53, (C) 1999 OSA, Figure (b) reproduced from Ref. 87, (C) 2002 OSA, Figures (c)-(g) adapted from Ref. 59, (c) 2010 OSA.

Similar-looking geometries have been conceived using metals. However, the propagating channel waveguide plasmons supported by these pillars, slits or holes possess smaller effective wavelengths and allow the structure to be thinner ${ }^{57-60}$. Also, the excited waveguide modes have propagation constants which sensitively depend on the dimensions of the slits or pillars, Figures $3 \mathrm{c}-\mathrm{g}$. This sensitivity constitutes an excellent means to tailor the phase of the transmitted field depending on a locally varying geometrical parameter. As shown in Figure 4, variation of the slit widths, between $80 \mathrm{~nm}$ and $150 \mathrm{~nm}$, engraved in a $400 \mathrm{~nm}$ thick gold film, could be used to form a one-dimensional plasmonic microlens ${ }^{58}$. The size of the lens is $3 \mu \mathrm{m}$ and realizes a focal spot at a distance of $5.3 \mu \mathrm{m}$ for visible light of $637 \mathrm{~nm}$ wavelength. The waveguide model was also used in the design of two-dimensional pillar-based mircolenses ${ }^{59}$, Figures $3 \mathrm{c}-\mathrm{g}$. There, the light propagates along the interspaces between the individual gold columns. The geometrical details of the structures 
were modified to affect the phase of the transmitted light in a predefined manner. Specifically, the aim was to realize the phase function of a lens

$$
\varphi(x, y)=\frac{2 \pi f}{\lambda}-\frac{2 \pi \sqrt{f^{2}+x^{2}+y^{2}}}{\lambda},
$$

where $f$ is the focal length and $\lambda$ the design wavelength.
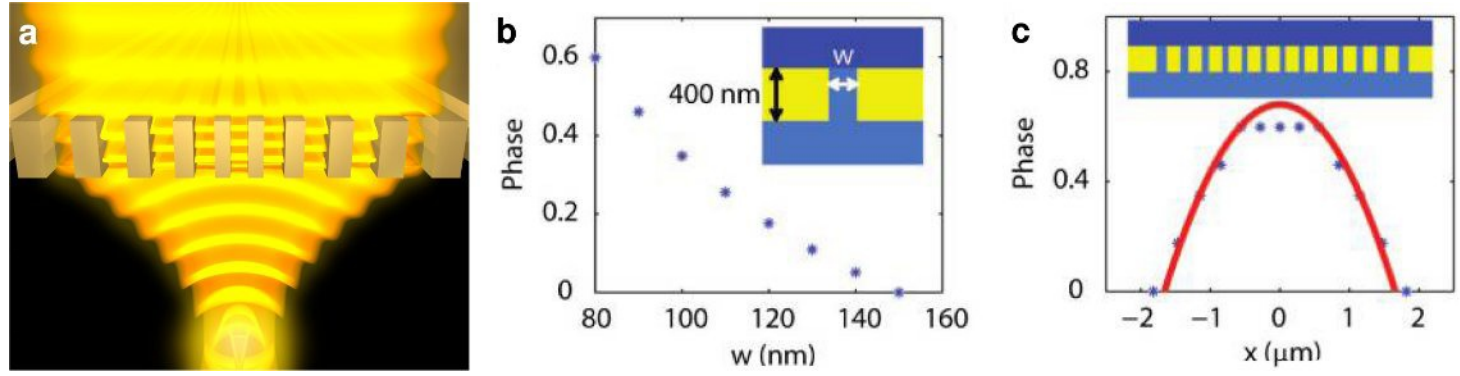

Figure 4. (a) Planar cylindrical lens formed by an array of slits in a metallic substrate. (b) The slit width determines the phase delay experienced by the channel-waveguided light. (c) A parabolic phase front is established, achieving focusing in the near-field at a distance of $5.3 \mathrm{~mm}$ behind the lens for a wavelength of $637 \mathrm{~nm}$. Figures (b) and (c) adapted from Ref. 58, (C) 2009 ACS.

Clearly, relying on propagation effects these devices do not impress with a minimum in thickness when compared to the planar structures discussed in the previous section. Therefore, the overall efficiency can be higher here. The design of the unit cells can rely on a huge backbone of results that were obtained when discussing enhanced transmission through subwavelength nanoapertures. For example, it is well known that coaxial or cross-shaped apertures sustain a much better incoupling efficiency when compared to simple square or rectangular apertures. With reported results for the transmission efficiency as high as $80 \%$ and more, devices with high efficiency, as desired for lenses, are more likely in reach $^{61}$. In the end, a specific application requires a trade-off between the disparate goals in integration, flatness, and compactness on the one hand, and efficiency on the other hand. We foresee that engineering this trade-off will likely be a dominating effort once the scientific aspects of these metamaterial elements are mastered.

a

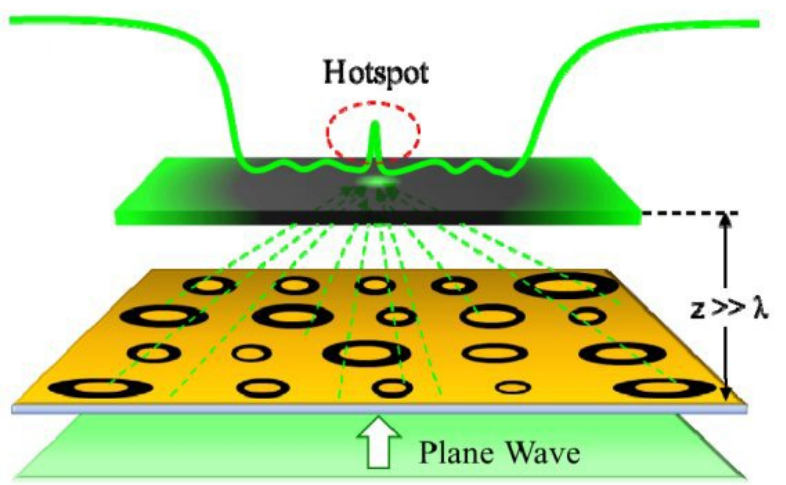

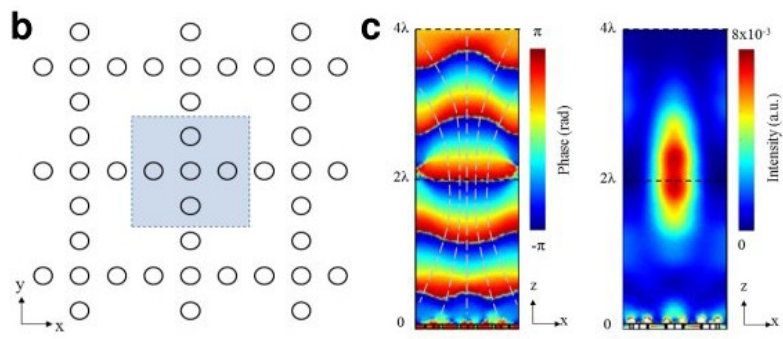

Figure 5. (a) Metamaterial aperture arrays designed to create super-oscillatory hotspots in a plane behind the structure. (b)(d) Arranging the ring apertures in a sparsed-out array breaks the symmetry and allows different local phase delays resulting in a curvature of the transmitted light. Figures adapted from Ref. 64, (C) 2013 OSA.

Beyond new, miniaturized lens designs, metamaterials may also provide lenses with focusing capabilities beyond the diffraction limit. As a step in this direction, a nanohole array in a thin metallic screen has been reported to produce focal spots with dimensions much smaller than the wavelength ${ }^{62-64}$, Figure 5. It exploits a phenomenon known as superoscillation, which allows band-limited functions to oscillate locally arbitrarily fast ${ }^{65}$, i.e. having a spatial modulation that can be much smaller than half the wavelength. It comes, however, usually at the expense of large side lobes ${ }^{66}$. A metallic 
screen lens with a diameter of $40 \mu \mathrm{m}$ was created for imaging purposes, which delivered a resolution sufficient to identify objects with a size of $\lambda / 3$ and to even separate them optically down to distances of $\lambda / 6$ and better. The superoscillatory lens may find application as a super-resolution microscope ${ }^{67}$.

\section{IMPLEMENTATION OF COMPLEX TRANSMISSION FUNCTIONS}

Beyond pure focusing and imaging tasks, metamaterials can also be employed in more general wave-shaping devices. For some optical elements, the desired transmission (or reflection) function is not analytically known as compared to a lens, Eq. (3), or a grating. Besides a rather complex phase function also a sophisticated amplitude distribution may be exploited - possibly in conjunction. This is the realm of holograms, which are typically designed to produce complicated wave fronts. These wave fronts might be shaped such that they look as if they would correspond to scattered light from a three-dimensional object. This type of holography found its way also into a specific direction of art. There, from a thin sheet of material into which the hologram is inscribed emerges the illusion of an entire object or even complicated scenes. But unlike holograms made in the earlier days, where holograms were recorded by interference of object beam and reference beam, now, with the method of computer-generated holography, the hologram's transmission function (amplitude or phase, or both) can be designed by a numerical algorithm and then implemented by micro- or nanostructuring techniques. A physical representation of the object is no longer necessary and a digital model or image is sufficient. This enables holograms for more abstract wave fields, i.e. those representing data stored in a binary fashion ${ }^{68}$. The advantage lies in the enormous flexibility of this approach. The first CGH was an amplitude hologram working with the detour phase principle, already allowing for simultaneous amplitude and phase modulation, affecting, however, the first-order diffracted light, not the zeroth order. It was printed with a plotter and then scaled down using photographic techniques ${ }^{69,70}$. The transition to dielectric holograms produced with lithography allowed for phase-only modulation of light with its beneficial impact on the energetic efficiency of the devices.

With metamaterials, another class of building blocks for CGHs became available that not only provides phase and amplitude modulation $^{71}$, but which also comes up with interesting spectral properties ${ }^{72}$. For an infinite, two-dimensional array of identical meta-atoms, i.e. a homogeneous metasurface, the transmitted (or reflected) wave remains a plane wave in the far-field if the periodicity of the meta-atoms is sub-wavelength. Tuning the shape of the meta-atoms allows for adjusting amplitude and phase of the far-field wave. By assembling finite-sized patches of different metamaterials a CGH can be composed whose inhomogeneous structure produces a complicated wave front. The assignment of what exact meta-atom shape needs to be used at a certain location (pixel) of the $\mathrm{CGH}$, is an inversion problem that is usually solved by an iterative algorithm, e.g. Iterative Fourier Transform Algorithm (IFTA) ${ }^{73,74}$. The algorithm needs to be fed with a database of available meta-atoms and their impact on the light's phase and amplitude. This database can be acquired by numerical means, such as full wave finite-difference time-domain method simulations or rigorous coupled wave analysis.

With the pixel-based approach and by applying several layers of gold elements with different shapes, it was possible to obtain a large range of phase manipulation which was used to encode a metamaterial CGH for image projection ${ }^{75}$. The specific hologram, as seen in Figure 6, was operated off-resonance of the plasmon-polaritons at a design wavelength of $10.6 \mu \mathrm{m}$, resulting in only small absorption losses. Hence, in this case the effect of amplitude modulation could be neglected in good approximation in the CGH encoding algorithm. Similarly, the phase effect provided by V-shaped antennas has been suggested in a CGH design at telecom wavelengths ${ }^{76}$. However, for metamaterials operating near their resonance, amplitude and phase effects are intrinsically entangled. In particular, the resonances are accompanied by the strongest absorption but they correspond also to the spectral domain of highest dispersion, leading to the largest phase modulations.

A metamaterial with a spectrum synthesized from electric and magnetic resonances is the fishnet structure ${ }^{77,78}$, Figures $6 \mathrm{~d}$ and e. In a numerical study, this metamaterial geometry has been shown to offer a wide range of wavelength-sensitive transmission values (real and imaginary part, i.e. comprising amplitude and phase) which can be used to encode holograms in the visible or near infrared ${ }^{79,71}$. Unlike the $\mathrm{V}$-antenna designs, though, the range of phase delays imposed by a single fishnet metal-dielectric-metal layer stack turned out to be significantly smaller than the desired $2 \pi^{71}$. Nevertheless, even with reduced coverage of the complex transmission coefficient, Figure $6 \mathrm{f}$, it is possible to encode a hologram, Figure $6 \mathrm{~g}$. By stacking multiple layers, the available phase values can cover the full range of $2 \pi$, but at the same time the transmitted amplitude goes down. Thus, the original benefit of accessing the full $2 \pi$, which is the capability to deflecting light at will in any region of the image plane and therefore achieve energy-efficient CGHs, is annihilated. 
In the extreme case of binary holograms, e.g. low/high amplitude or with two phase levels at constant amplitude, diffraction will occur symmetrically. This limits the freedom of distributing the diffracted light at will across the full transmittance hemisphere or the full image plane. Therefore, when defining a confined target image area (signal window), as conceptually shown in Figure $6 \mathrm{~g}$, there will always be a twin image, effectively wasting half of the incidence light. In addition, if the sum of the complex transmission values across the CGH is non-vanishing, as in the CGHs in Ref. 71, Figure 6f,g, there will be a central zero-order peak in the far field (Fourier plane) and further energy is lost. In the numerical study in Ref. 71 about $82 \%$ of the transmitted light gathers in this central peak. However, just around $16 \%$ is transmitted in the first place, a similar problem as experienced by many devices discussed so far.
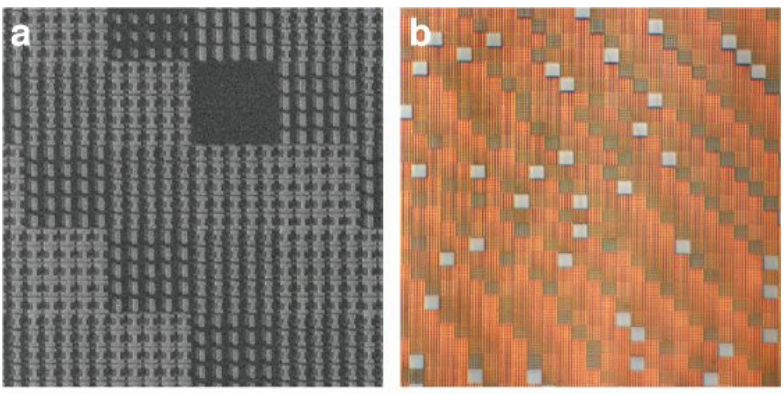

C

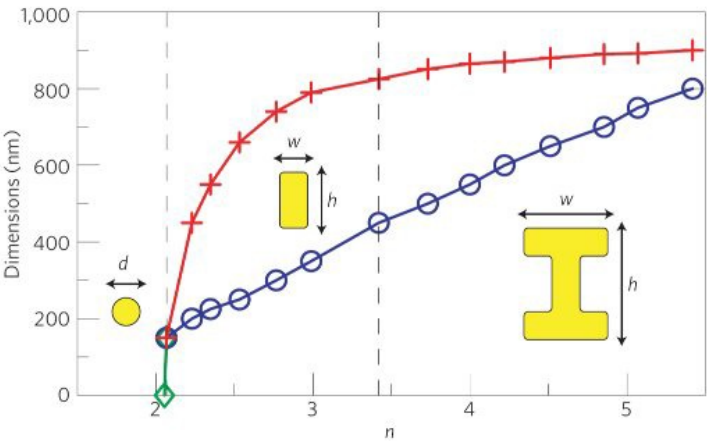

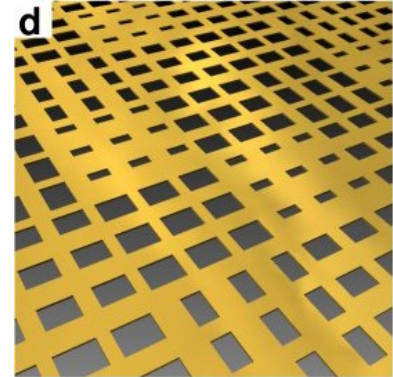
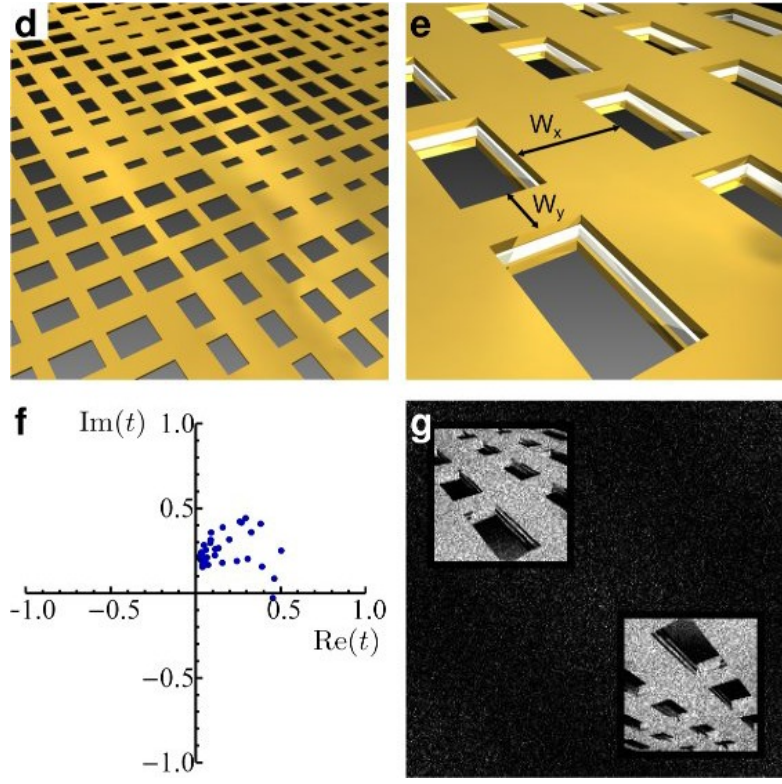

Figure 6. (a)-(c) Metamaterial CGH assembled from off-resonant circular, I-shaped and double-T-shaped gold elements in a stacked arrangement (three metallic layers). Each hologram pixel has a size of $5 \mu \mathrm{m}$ and acts as homogeneous material with specific refractive index $\mathrm{n}$. The index is adjusted by shape and dimensions of the unit cell's metal content. The operation wavelength is at $10.6 \mu \mathrm{m}$. (d)-(e) Hologram composed of resonant fishnet metamaterial where the pixels' responses are determined by the lateral widths $\mathrm{W}_{\mathrm{x}}, \mathrm{W}_{\mathrm{y}}$ of the metal bars. The unit cell size is $600 \mathrm{~nm}$. (f) Each of the tupels $\mathrm{W}_{\mathrm{x}}, \mathrm{W}_{\mathrm{y}}$ produces a specific transmission coefficient $\mathrm{t}$. (g) Simulated far-field intensity image of the CGH at a wavelength of $740 \mathrm{~nm}$. Figures (a)-(c) adapted from Ref. 75, (C) 2012 NPG, Figures (d)-(g) reproduced from Ref. 71, (C) 2011 AIP.

The incorporation of gain materials that are either optically or, more desirably, electrically pumped might offer one way to compensate direct absorption losses. Loss-compensated metamaterials based on the fishnet design have been presented experimentally by incorporating gain materials into the structure. A figure-of-merit, defined here as the ratio of absolute value of the real to imaginary part, as large as 26 has been reported and it has been speculated that for a suitable operation the figure-of-merit might be macroscopically large, i.e. being huge on a logarithmic scale ${ }^{80}$. However, whereas this incorporation of gain has been demonstrated with traditional metamaterials where all unit cells are identical, the compensation of absorption has not yet been demonstrated in the context of metasurfaces. Here, more research experimentally, theoretically and computationally - is necessary to fully understand the intricate interplay between gain and observable dispersive effects to take advantage of the existing technology $\mathrm{y}^{81,82}$.

In this regard, binary holograms might be the logical starting point and an important test class of CGHs. Besides, they are especially robust against fabrication tolerances, as the image formation relies on just two distinct pixel types within the CGH. This allows for using rather exotic materials to form the hologram, e.g. carbon nanotubes ${ }^{83}$. As long as the optical responses of the two pixel types are different, the image is reproduced. This makes binary holograms also work across an extended spectral domain, accompanied only by changes in the angular size of the projected image and in the obtained energetic efficiencies for different wavelengths. 


\section{MULTI-WAVELENGTHS OPERATION}

To obtain holograms that deliver truly distinct wave fronts or images at different wavelengths, the most obvious idea would be to have the pixels of the hologram produce a wavelength-specific response. This is difficult to accomplish with dielectrics, and thus several back-door techniques have been developed. For example, height profiles with extreme aspect ratios were built, where on a CGH for wavelength $\lambda_{1}$ multiples of a $2 \pi$ phase delay were added pixelwise on top to adjust the response for a second wavelength $\lambda_{2}{ }^{84}$. Alternatively, the polarization state could be used as a distinguishing and exploitable criterion for the second wavelength. Creating birefringent hologram pixels with the effective medium approach, as seen in Figure 3b, two-color holograms were realized ${ }^{85-87}$. It has also been demonstrated that the use of a pixelated stack of wavelength-selective mirrors is suitable to form multi-wavelength CGHs for reflection ${ }^{88}$.

Even more obvious is the canonical approach of using different color channels that can be encoded at laterally separated parts of the $\mathrm{CGH}$, which are then also illuminated by spatially separated beams of different wavelength ${ }^{89}$. An ordinary CGH can also be tricked into multi-color mode by exploiting the different diffraction angles observed for the different colors. Overlaying the proper parts of each color channel in the signal window and blocking all the unwanted light creates the desired color image ${ }^{90}$. Furthermore, for Fresnel-type holograms the different wavelengths experience different planes of focus, providing an additional degree of manipulation freedom ${ }^{91}$.

Surely, expanding the two-dimensional holographic structure into the third dimension adds new degrees of freedom in abundance. So-called volume holograms are a well-known field on their own, capable of delivering spectrally or angularly multiplexed wave fronts ${ }^{92,93}$. The holographic volume does not have to be continuous, though. It is possible, and existing fabrication constraints compelled to do so, to arrange a series of distinct two-dimensional hologram layers behind each other. In this setup the dispersive information is aggregated by free-space diffraction during propagation between the layers ${ }^{94}$.

However, in order to encode multi-wavelength information in a single, thin two-dimensional layer, true dispersion control of the material itself is required. This is where plasmonic metamaterials with their pronounced dispersive behavior have strong potential. While classic dielectric materials are inherently non- or weakly dispersive, the transmissive and reflective properties, in amplitude and phase, of metamaterials depend sensitively on the wavelength of light. Modifications in the metamaterial geometry allow for shifting and shaping the plasmonic resonances. Thus, not only adjustments of the transmission values at a single wavelength turn out to be feasible, but the design of an entire spectral response can be envisioned.

A first step in this direction was recently proposed with the generation of a dual-wavelength hologram based on a thin metasurface $^{72}$ as shown in Figure 7a. Here, again the lateral size parameters of a fishnet metamaterial were used for tuning, this time in order to vary the spectral transmission behavior. From a selected set of such fishnet versions a CGH was constructed with the help of a special coding scheme, Figures $7 \mathrm{~d}$ and e. The resulting CGH acts as a binary hologram at each of the two infrared wavelengths $905 \mathrm{~nm}$ and $1385 \mathrm{~nm}$, projecting distinct and independent images in the Fourier plane, Figures $7 \mathrm{~b}$ and c. The energetic efficiencies are very low, though, in the range of one tenth of a percent, due to weak transmission. Nevertheless, extrapolating the possibilities of simultaneous spatial and spectral light control to more than two and ultimately to a continuum of wavelengths, light-bullet shaping might become viable without spectral separation workarounds ${ }^{95}$.

Other applications for such CGHs lie in the context of multiplexed data storage. Gold nanorods, for example, exhibit a plasmon resonance at a wavelength depending on their length and orientation with respect to the illumination polarization. Hence, an existing mixture of nanorods with random sizes and aspect ratios can be reshaped very selectively by an intense laser pulse ${ }^{68}$. Writing a pattern in such a nanorod sample is a way to store wavelength- as well as polarization-multiplexed information at the same time. This information can later be read by the same resonancespecific response mechanism. Likewise, the spectral selectivity of plasmonic nanoparticles can be used as a key to create distinctive color impressions for illumination with white light ${ }^{96}$ or entangle polarization and color ${ }^{97}$.

For some applications, however, a constant broadband behavior is favored over a significantly dispersive device response. The phenomenon of anomalous refraction can be applied broadband, as demonstrated for dipole antennas and the construction of a vortex beam in the range of $670 \mathrm{~nm}$ to $1100 \mathrm{~nm}^{98}$. Similarly, combining two sub-units of V-antenna arrays it was possible to create a broadband quarter-wave plate for the infrared ${ }^{99}$, Figure $7 \mathrm{f}$. Here, due to the superposition of the symmetric and anti-symmetric mode, not only a spectrally broad resonance could be achieved for the V-antenna, but also the phase response of this superposition turned out to be nearly linear over a large spectral range. What is more, each sub-unit with its balanced tandem unit cell produces the same distortions in their scattering response, resulting in a robust circular polarization state in the spectral range from 5 to $8 \mu \mathrm{m}$. 

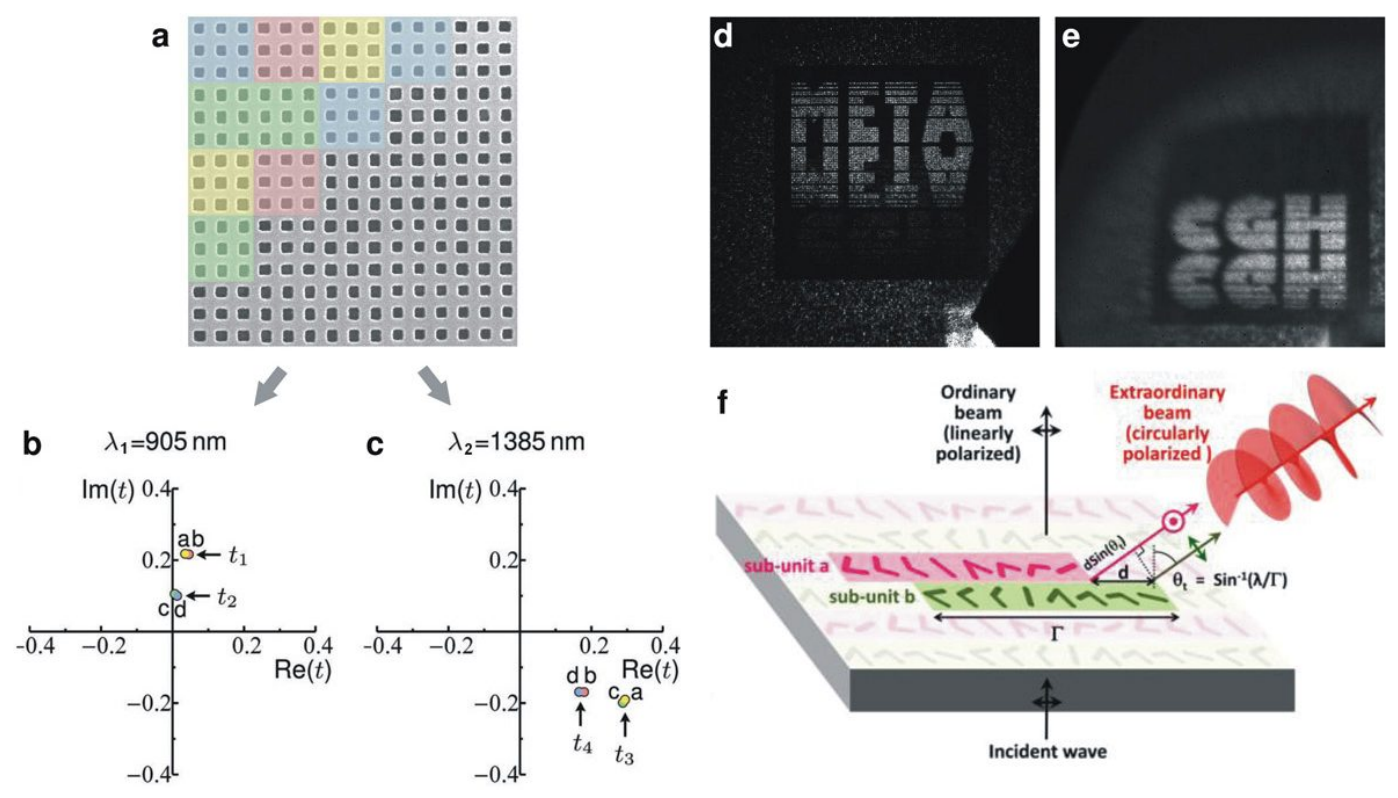

Figure 7. (a) CGH composed of fishnet metamaterial to produce a wavelength-specific response in form of two different images at wavelengths $905 \mathrm{~nm}$ and $1385 \mathrm{~nm}$. (b) and (c) Four different fishnet versions, labeled 'a', 'b', 'c' and 'd' form a special constellation of interchanging pairs regarding their transmission coefficients at the two wavelengths. (d) and (e) Holographically projected images at $905 \mathrm{~nm}$ and $1385 \mathrm{~nm}$, respectively. (f) A metamaterial-based quarterwave plate for broadband operation. Excited with linearly polarized light the two sub-units forming the metasurface generate two copropagating orthogonally polarized beams with a phase shift of $\pi / 2$ with respect to each other. The element is tailored such that a symmetric (S) and an antisysmmetric (A) oscillation mode interfere to form spatially separated (S-A) and $(\mathbf{S}+\mathbf{A})$ beams. The exploited extraordinary (S-A) beam provides a broadband and background-free circularly polarized light source for wavelengths between $5 \mu \mathrm{m}$ and $8 \mu \mathrm{m}$. Figures (a)-(e) adapted from Ref. 72 C) 2012 WILEY, Figure (f) reproduced from Ref. 99 (C) 2012 ACS.

\section{SUMMARY AND PERSPECTIVE}

In summary, the research on diffractive optical elements made from metamaterials appears as a nascent and burgeoning field. Metasurfaces are a further advancement of the realm of metamaterials towards devices with immediate relevance for various technology driven applications.

The key idea that triggered the development is the liberation from the constraint to work towards materials that act as bulk effective media. These materials, by definition, are required to consist of identical unit cells that are periodically or amorphously arranged in space. While relying on the strong interaction of carefully designed unit cells that usually contain metallic constituents, the reflection and transmission from slabs of such materials or from individual functional layers could be tailored with an extraordinary degree of freedom. Lifting the restriction of identical unit cells opened ways to purposefully shape light fronts upon requests for a given illumination. If the promise of metamaterials had been to provide independent control over permittivity and permeability, this promise can be reformulated in the domain of metasurfaces to provide independent control over the local amplitude, phase, and also the polarization of light.

This review showed the beginnings of carrying through on these promises. There is no need to encode over-complicated wave fronts in these proof-of-principle experiments. Replicating well-known examples from the context of classical optics and giving them an additional twist is fully sufficient in these studies. We have witnessed a large number of works where ultra-thin metasurfaces imprinted an abrupt phase gradient on an incident wave field to deflect light or create a spherical wave front to focus or diverge light; both properties equally across extended spectral domains. Such functionalities are valuable since they are immediately perceived, constitute an easy to formulate functionality, and already disclose the advantages of metasurfaces over classical optical elements.

Yet, it remains an ongoing challenge to fully utilize the proclaimed freedom and identify and realize further kinds of favorable wave fronts which were previously inaccessible. Herein lies a great potential for groundbreaking innovations and applications. 
Metasurfaces possess tremendous advantages such as the ultra-compactness of the devices, the possibility for either broadband or wavelength-dependent operation, the ability to achieve super-resolution and super-focusing, minimized image distortion and aberrations as well as the capability to control the outgoing polarization state ${ }^{100,101}$. As shown, implementations of flat, broadband lenses with reduced aberration, vortex beams, axicons, flat prisms with arbitrary directions of deflection, quarter-wave plates and holograms benefit from these advantages. Concepts developed in the world of diffractive optics and computer-generated holograms can be adapted for their design.

However, in the long run some essential problems for the further development of metasurfaces have to be solved. First, it remains desirable to have a feasible material platform available to address the entire complex plane in transmission or reflection, that is, the full phase range of $2 \pi$ and complete amplitude range 0 to 1 . This, for sure, remains an idealistic goal since dispersion and absorption are intimately linked and their simultaneous appearance is unavoidable. Maybe, by incorporating resonant dielectric-only nanoantennas whose losses are almost only of radiative nature, these problems

might be mitigated ${ }^{102-104}$. Recent major advances in the field of all-dielectric metasurfaces indicate that many application needs might be fulfilled much better by nanostructured high-index dielectrics than by metallic nanostructures. As an example, we have recently realized a so-called Huygens' surface based on dielectric nanostructures, which allows for phase control without sacrificing detrimental losses ${ }^{104}$. However, to make full use of dielectric metasurfaces it remains to develop a versatile material and technology platform for a high-index, low-loss dielectric in the visible spectral domain, the nanostructures of which support complexly shaped resonant excitations.

Secondly, the design of holograms made from metamaterials would benefit from an advancement and adaptation of corresponding design tools. Nowadays, the assumption is usually that the unit cells' geometries vary smoothly across the surface such that the local transmission is well approximated by what the transmission of the same unit cell would be when embedded in a periodic lattice. Moreover, full-wave simulations for the design of CGHs can only be performed as long as the metasurface consists of periodically arranged super cells that have a manageable size which does not conflict with available computational resources. Both aspects constitute a limitation. Design tools that properly take the potential nonlocal response of the unit cells into account are needed. They shall predict the optical response of a metamaterial hologram that is large in size (compared to the wavelength), but which is assembled from highly resonant, complicated, and disparate sub-wavelength unit cells.

Finally, motivated by an advancement of technologies diffractive elements based on metasurfaces should eventually be incorporated into actual devices. They shall not just be studied on their own, but the functionality they provide shall make a difference in an actual application. The structures that can be provided generally act as multi-functional elements capable of transforming complicated optical devices into ultra-thin, ultra-light, portable components. Such elements might be incorporated in the design of optical circuits ${ }^{105-107}$, used for optical measurements and beam analysis ${ }^{108}$, optical particle control ${ }^{109}$ and visual applications ${ }^{110}$. Dynamic tunability of the devices would be highly desirable and would greatly enhance the applicability of metasurfaces.

With all these aspects and potential benefits it appears natural to consider metasurfaces as a route towards unprecedented real-world optical devices.

\section{ACKNOWLEDGMENTS}

The authors would like to acknowledge support by the Federal Ministry of Education and Research (PhoNa), the State of Thuringia within the Pro-Excellence program (MeMa and ACP 2020), as well as the Carl Zeiss Foundation.

\section{REFERENCES}

[1] Yu, N. and Capasso, F., "Flat optics with designer metasurfaces," Nature Materials 13, 139 (2014).

[2] Kildishev, A.V., Boltasseva, A., and Shalaev, V. M., "Planar photonics with metasurfaces," Science 339, 6125 (2013).

[3] Barber, D. J. and Freestone, I. C., "An investigation of the origin of the colour of the lycurgus cup by analytical transmission electron microscopy," Archaeometry 32, 33-45 (1990).

[4] Mie, G., "Beiträge zur Optik trüber Medien, speziell kolloidaler Metallösungen," Annalen der Physik, Vierte Folge, Band 25, No. 3: 377-445 (1908).

[5] Rayleigh, L. "XLII: On the scattering of light by a cloud of similar small particles of any shape and oriented at random," Philosophical Magazine Series 6; 35(209), 373-381 (1918). 
[6] Faraday, M., "Experimental relations of gold (and other metals) to light," Philos. Trans. R. Soc. London 147, 145181 (1857).

[7] Luk'yanchuk, B., Zheludev, N. I., Maier, S. A., Halas, N. J., Nordlander, P., Giessen, H., and Chong, C. T., "The Fano resonance in plasmonic nanostructures and metamaterials," Nature Materials 9, 707-715 (2010).

[8] Pendry, J. B., "Negative Refraction Makes a Perfect Lens," Phys. Rev. Lett. 85, 3966-3969 (2000).

[9] Pendry, J. B., Schurig, D., and Smith, D. R., "Controlling electromagnetic fields," Science 312, 1780-1782 (2006).

[10] Schurig, D., Mock, J. J., Justice, B. J., Cummer, S. A., and Pendry, J. B., Starr, A. F., and Smith, D. R., "Metamaterial electromagnetic cloak at microwave frequencies," Science 314, 977-980 (2006).

[11] Chen, H., Chan, C. T., and Sheng, P., "Transformation optics and metamaterials," Nature Materials 9, 387-396 (2010).

[12] Liu, N., Mesch, M., Weiss, T., Hentschel, M., and Giessen, H. "Infrared perfect absorber and its application as plasmonic sensor," Nano Lett. 10, 2342-2348 (2010).

[13] Hedayati, M. K., Javaherirahim, M., Mozooni, B., Abdelaziz, R., Tavassolizadeh, A., Chakravadhanula, V. S. K., et al., "Design of a perfect black absorber at visible frequencies using plasmonic metamaterials," Advanced Materials 23, 5410-5414 (2011).

[14] Liu, N., Hentschel, M., Weiss, T., Alivisatos, A.P., and Giessen, H., "Three-dimensional plasmon rulers," Science $332,1407-1410$ (2011).

[15] Decker, M., Klein, M. W., Wegener, M., and Linden, S., "Circular dichroism of planar chiral magnetic metamaterials," Optics Lett. 32, 856-858 (2007).

[16] Plum, E., Fedotov, V. A., Schwanecke, A. S., Zheludev, N. I., and Chen, Y., "Giant optical gyrotropy due to electromagnetic coupling," Appl. Phys. Lett. 90, 223113 (2007).

[17] Helgert C, Pshenay-Severin E, Falkner M, Menzel C, Rockstuhl C, Kley E-B, et al. Chiral Metamaterial Composed of Three-Dimensional Plasmonic Nanostructures. Nano Lett. 2011; 11: 4400-4404.

[18] Zhao Y, Alù A. Manipulating light polarization with ultrathin plasmonic metasurfaces. Phys. Rev. B 2011; 84: 205428.

[19] Menzel C, Helgert C, Rockstuhl C, Kley E-B, Tünnermann A, Pertsch T, Lederer F. Asymmetric Transmission of Linearly Polarized Light at Optical Metamaterials. Phys. Rev. Lett. 2010; 104: 253902.

[20] Valentine J, Zhang S, Zentgraf T, Ulin-Avila E, Genov DA, Bartal G, Zhang X. Three-dimensional optical metamaterial with a negative refractive index. Nature 2008; 455: 376-379.

[21] Soukoulis MC, Wegener M. Past achievements and future challenges in the development of three-dimensional photonic metamaterials. Nature Photonics 2011; 5: 523-530.

[22] Boltasseva A, Shalaev VM. Fabrication of optical negative-index metamaterials: Recent advances and outlook. Metamaterials 2008; 2: 1-17.

[23] Radke A, Gissibl T, Klotzbücher T, Braun PV, Giessen H. Three-Dimensional Bichiral Plasmonic Crystals Fabricated by Direct Laser Writing and Electroless Silver Plating. Advanced Materials 2011; 23: 3018-3021.

[24] Chanda D, Shigeta K, Gupta S, Cain T, Carlson A, Mihi A, et al. Large-area flexible 3D optical negative index metamaterial formed by nanotransfer printing. Nature Nanotechnology 2011; 6: 402-407.

[25] Fan JA, Wu C, Bao K, Bao J, Bardhan R, Halas NJ, et al. Self-assembled plasmonic nanoparticle clusters. Science 2010; 328: 1135-1138.

[26] Mühlig S, Cunningham A, Scheeler S, Pacholski C, Burgi T, Rockstuhl C, Lederer F. Self-Assembled Plasmonic Core-Shell Clusters with an Isotropic Magnetic Dipole Response in the Visible Range. ACS Nano 2011; 5: 65866592.

[27] Menzel C, Paul T, Rockstuhl C, Pertsch T, Tretyakov S, Lederer F. Validity of effective material parameters for optical fishnet metamaterials. Phys. Rev. B 2010; 81: 035320.

[28] Decker M, Staude I, Falkner M, Dominguez J, Neshev DN, Brener I, et al. High-Efficiency Dielectric Huygens' Surfaces. Advanced Optical Materials 2015; 3: 813.

[29] Yu N, Genevet P, Kats MA, Aieta F, Tetienne JP, Capasso F, Gaburro Z. Light Propagation with Phase Discontinuities: Generalized Laws of Reflection and Refraction. Science 2011; 334: 333-337.

[30] Aieta F, Genevet P, Yu N, Kats MA, Gaburro Z, Capasso F. Out-of-Plane Reflection and Refraction of Light by Anisotropic Optical Antenna Metasurfaces with Phase Discontinuities. Nano Lett. 2012; 12: 1702-1706.

[31] Balanis CA. Antenna Theory: Analysis and Design. Hoboken, NJ: John Wiley \& Sons, Inc., 2005.

[32] Bharadwaj P, Deutsch B, Novotny L. Optical Antennas. Advances in Optics and Photonics 2009; 1: 438-483.

[33] Novotny L, van Hulst N. Antennas for light. Nature Photonics 2011; 5: 83-90. 
[34] Petschulat J, Menzel C, Chipouline A, Rockstuhl C, Tünnermann A, Lederer F, Pertsch T. Multipole approach to metamaterials. Phys. Rev. A 2008; 78: 043811.

[35] Papakostas A, Potts A, Bagnall DM, Prosvirnin SL, Coles HJ, Zheludev NI. Optical Manifestations of Planar Chirality. Phys. Rev. Lett. 2003; 90: 107404.

[36] Prosvirnin SL, Zheludev NI. Polarization effects in the diffraction of light by a planar chiral structure. Phys. Rev. E 2005; 71: 037603.

[37] Blanchard R, Aoust G, Genevet P, Yu N, Kats MA, Gaburro Z, Capasso F. Modeling nanoscale V-shaped antennas for the design of optical phased arrays. Phys. Rev. B 2012; 85: 155457.

[38] Capasso F, "Classical optics in a new light: flat photonics with metasurfaces I", Presentation held at MPNS COST Action Training School - MP1204 TERA-MIR Radiation: Materials, Generation, Detection and Applications. Cortona; May 21, 2013.

[39] Genevet P, Yu N, Aieta F, Lin J, Kats MA, Blanchard R, et al. Ultra-thin plasmonic optical vortex plate based on phase discontinuities. Appl. Phys. Lett. 2012; 100: 013101.

[40] Ni X, Emani NK, Kildishev AV, Boltasseva A, Shalaev VM. Broadband Light Bending with Plasmonic Nanoantennas. Science 2012; 335: 427.

[41] Aieta F, Genevet P, Kats MA, Yu N, Blanchard R, Gaburro Z, Capasso F. Aberration-free ultra-thin flat lenses and axicons at telecom wavelengths based on plasmonic metasurfaces. Nano Lett. 2012; 12: 4932-4936.

[42] Information from website; http://www.osaopn.org/home/newsroom/2012/august/metasurface_makes_ultrathin_lens_aberration-free/\#.UlEvo5zjCB4; Date of citation: August 4, 2015.

[43] Naik GV, Schroeder JL, Ni X, Kildishev AV, Sands TD, Boltasseva A. Titanium nitride as a plasmonic material for visible and near-infrared wavelengths. Opt. Mater. Express 2012; 2: 478-489.

[44] West PR, Ishii S, Naik GV, Emani NK, Shalaev VM, Boltasseva, A. Searching for better plasmonic materials. Laser \& Photonics Reviews 2010; 4: 495-808.

[45] Kats MA, Genevet P, Aoust G, Yu N, Blanchard R, Aieta F, et al. Giant birefringence in optical antenna arrays with widely tailorable optical anisotropy. PNAS 2012; 109: 12364-12368.

[46] Chen X, Huang L, Mühlenbernd H, Li G, Bai B, Tan Q, et al. Dual-polarity plasmonic metalens for visible light. Nature Communications 2012; 3: 1198.

[47] Chen X, Huang L, Mühlenbernd H, Li G, Bai B, Tan Q, et al. Reversible Three-Dimensional Focusing of Visible Light with Ultrathin Plasmonic Flat Lens. Adv. Optical Mater. 2013; 1: 517-521.

[48] Kang M, Feng T, Wang H-T, Li J. Wave front engineering from an array of thin aperture antennas. Optics Express 2012; 20: 15882-15890.

[49] Monticone F, Estakhri NM, Alù A. Full Control of Nanoscale Optical Transmission with a Composite Metascreen. Phys. Rev. Lett. 2013; 110: 203903.

[50] Clausnitzer T, Kämpfe T, Kley E-B, Tünnermann A, Peschel U, Tishchenko AV, Parriaux O. An intelligible explanation of highly-efficient diffraction in deep dielectric rectangular transmission gratings. Optics Express 2005; 13: 10448-10456.

[51] Farn MW. Binary gratings with increased efficiency. Applied Optics 1992; 31: 4453-4458.

[52] Chen FT, Craighead HG. Diffractive lens fabricated with mostly zeroth-order gratings. Optics Letters 1996; 21 : 177-179.

[53] Lalanne P, Astilean S, Chavel P, Cambril E, Launois H. Design and fabrication of blazed binary diffractive elements with sampling periods smaller than the structural cutoff. JOSA A 1999; 16: 1143-1156.

[54] Mait JN, Prather DW, Mirotznik MS. Design of binary subwavelength diffractive lenses by use of zeroth-order effective-medium theory. JOSA A 1999; 16: 1157-1167.

[55] Freese W, Kämpfe T, Kley E-B, Tünnermann A. Design of binary subwavelength multiphase level computer generated holograms. Optics Letters 2010; 35: 676-678.

[56] Fattal D, Li J, Peng Z, Fiorentino M, Beausoleil RG. Flat dielectric grating reflectors with focusing abilities. Nature Photonics 2010; 4: $466-470$.

[57] Shi H, Wang C, Du C, Luo X, Dong X, Gao H. Beam manipulating by metallic nano-slits with variant widths. Optics Express 2005; 13: 6815-6820.

[58] Verslegers L, Catrysse PB, Yu Z, White JS, Barnard ES, Brongersma ML, Fan S. Planar Lenses Based on Nanoscale Slit Arrays in a Metallic Film. Nano Lett. 2009; 9: 235-238.

[59] Verslegers L, Catrysse PB, Yu Z, Shin W, Ruan Z, Fan S. Phase front design with metallic pillar arrays. Optics Letters 2010; 35: 844-846. 
[60] Bozhevolnyi SI, Volkov VS, Devaux E, Laluet J-Y, Ebbesen TW. Channel plasmon subwavelength waveguide components including interferometers and ring resonators. Nature 2006; 440: 508-511.

[61] Lin L, Goh XM, McGuinness LP, Roberts A. Plasmonic Lenses Formed by Two-Dimensional Nanometric CrossShaped Aperture Arrays for Fresnel-Region Focusing. Nano Lett. 2010; 10: 1936-1940.

[62] Huang FM, Zheludev N, Chen Y, de Abajo FJG. Focusing of light by a nanohole array. Appl. Phys. Lett. 2007; 90: 091119.

[63] Huang FM, Kao TS, Fedotov VA, Chen Y, Zheludev NI. Nanohole Array as a Lens. Nano Lett. 2008; 8: 24692472.

[64] Roy T, Rogers ETF, Zheludev NI. Sub-wavelength focusing meta-lens. Optics Express 2013; 21: 7577-7582.

[65] Berry VM, Popescu S. Evolution of quantum superoscillations and optical superresolution without evanescent waves. Journal of Physics A: Mathematical and General 2006; 39: 6965-6977.

[66] Huang FM, Zheludev NI. Super-Resolution without Evanescent Waves. Nano Lett. 2009; 9: 1249-1254.

[67] Rogers ETF, Lindberg J, Roy T, Savo S, Chad JE, Dennis MR, Zheludev NI. A super-oscillatory lens optical microscope for subwavelength imaging. Nature Materials 2012; 11: 432-435.

[68] Zijlstra P, Chon JWM, Gu M. Five-dimensional optical recording mediated by surface plasmons in gold nanorods. Nature 2009; 459: 410-413.

[69] Brown BR, Lohmann AW. Complex Spatial Filtering with Binary Masks. Applied Optics, 1966; 5: 967-969.

[70] Lohmann AW, Paris DP. Binary Fraunhofer Holograms, Generated by Computer. Applied Optics 1967; 6: 17391748.

[71] Walther B, Helgert C, Rockstuhl C, Pertsch T. Diffractive optical elements based on plasmonic metamaterials. Appl. Phys. Lett. 2011; 98: 191101.

[72] Walther B, Helgert C, Rockstuhl C, Setzpfandt F, Eilenberger F, Kley E-B, et al. Spatial and Spectral Light Shaping with Metamaterials. Adv. Mat. 2012; 24: 6300-6304.

[73] Wyrowski F, Bryngdahl O. Digital holography as part of diffractive optics. Rep. Prog. Phys. 1991; 54: 1481-1571.

[74] Gerchberg RW, Saxton WO. A practical algorithm for the determination of the phase from image and diffraction plane pictures. Optik 1972; 35: 237-246.

[75] Larouche S, Tsai Y-J, Tyler T, Jokerst NM, Smith DR. Infrared metamaterial phase holograms. Nature Materials 2012; 11: 450-454.

[76] Zhou F, Liu Y, Cai W. Plasmonic holographic imaging with V-shaped nanoantenna array. Optics Express 2013; 21: 4348-4354.

[77] Zhang S, Fan W, Malloy KJ, Brueck SR, Panoiu NC, Osgood RM. Near-infrared double negative metamaterials. Optics Express 2005; 13: 4922-4930.

[78] Zhang S, Fan W, Panoiu NC, Malloy KJ, Osgood RM, Brueck SRJ. Experimental Demonstration of Near-Infrared Negative-Index Metamaterials. Phys. Rev. Lett. 2005; 95: 137404.

[79] Matsui T, Nomura T, Miura A, Fujikawa H, Ikeda N, Tsuya D, et al. Wavefront control by stacked metal-dielectric hole array with variable hole shapes. Optics Express 2013; 21: 6153-6161.

[80] Xiao S, Drachev VP, Kildishev AV, Ni X, Chettiar UK, Yuan H-K, Shalaev VM. Loss-free and active optical negative-index metamaterials. Nature 2010; 466: 735-738.

[81] Wuestner S, Pusch A, Tsakmakidis KL, Hamm JM, Hess O. Overcoming Losses with Gain in a Negative Refractive Index Metamaterial. Phys. Rev. Lett. 2010; 105: 127401.

[82] Fang A, Koschny T, Soukoulis CM. Self-consistent calculations of loss-compensated fishnet metamaterials. Phys. Rev. B 2010; 82: 121102.

[83] Butt H, Montelongo Y, Butler T, Rajesekharan R, Dai Q, Shiva-Reddy SG, et al. Carbon Nanotube Based High Resolution Holograms. Advanced Materials 2012; 24: OP331-OP336.

[84] Barton I, Blair P, Taghizadeh MR. Dual-wavelength operation diffractive phase elements for pattern formation. Optics Express 1997; 1: 54-59.

[85] Xu F, Tyan R-C, Sun P-C, Fainman Y, Cheng C-C, Scherer A. Form-birefringent computer-generated holograms. Optics Letters 1996; 21: 1513-1515.

[86] Zeitner UD, Schnabel B, Kley E-B, Wyrowski F. Polarization Multiplexing of Diffractive Elements with MetalStripe Grating Pixels. Applied Optics 1999; 38: 2177-2181.

[87] Yu W, Konishi T, Hamamoto T, Toyota H, Yotsuya T, Ichioka Y. Polarization-Multiplexed Diffractive Optical Elements Fabricated by Subwavelength Structures. Applied Optics 2002; 41: 96-100.

[88] Kämpfe T, Kley E-B, Tünnermann A, Dannberg P. Design and fabrication of stacked, computer generated holograms for multicolor image generation. Applied Optics 2007; 46: 5482-5488. 
[89] Fienup JR, Goodman JW. New ways to make computer-generated color holograms. Nouvelle Revue d'Optique 1974; 5: 269.

[90] M Araiza E, S Guel S, C Sifuentes G, I de la Rosa V, G Miramontes de L. Multichannel image storage with application to color multiplexing. Revista mexicana de física 2008; 54: 100-103.

[91] Bengtsson J. Kinoforms Designed to Produce Different Fan-Out Patterns for Two Wavelengths. Applied Optics 1998; 37: 2011-2020.

[92] van Heerden PJ. Theory of Optical Information Storage in Solids. Applied Optics 1963; 2: 393-400.

[93] Gerke TD, Piestun R. Aperiodic volume optics. Nature Photonics 2010; 4: 188-193.

[94] Johnson RV, Tanguay Jr AR. Stratified volume holographic optical elements. Optics Letters 1988; 13: 189-191.

[95] Cundiff ST, Weiner AM. Optical arbitrary waveform generation. Nature Photonics 2010; 4: 760-766.

[96] Kumar K, Duan H, Hegde RS, Koh SCW, Wei JN, Yang JKW. Printing colour at the optical diffraction limit. Nature Nanotechnology 2012; 7: 557-561.

[97] Ellenbogen T, Seo K, Crozier KB. Chromatic Plasmonic Polarizers for Active Visible Color Filtering and Polarimetry. Nano Lett. 2012; 12: 1026-1031.

[98] Huang L, Chen X, Mühlenbernd H, Li G, Bai B, Tan Q, et al. Dispersionless Phase Discontinuities for Controlling Light Propagation. Nano Lett. 2012; 12: 5750-5755.

[99] Yu N, Aieta F, Genevet P, Kats MA, Gaburro Z, Capasso F. A Broadband, Background-Free Quarter-Wave Plate Based on Plasmonic Metasurfaces. Nano Lett. 2012; 12: 6328-6333.

[100] Gansel JK, Thiel M, Rill MS, Decker M, Bade K, Saile V, et al. Gold Helix Photonic Metamaterial as Broadband Circular Polarizer, Science 2009; 325: 1513-1515.

[101] Zhao Y, Belkin MA, Alù A. Twisted optical metamaterials for planarized ultrathin broadband circular polarizers. Nature Communications 2012; 3: 870.

[102] Yang Y, Kravchenko II, Briggs DP, Valentine J. All-dielectric metasurface analogue of electromagnetically induced transparency. Nature Communications 2014; 5: 5753.

[103] Lin D, Fan P, Hasman E, Brongersma ML. Dielectric gradient metasurface optical elements. Science 2014; 345 : 298.

[104] Decker, M., Staude, I., Falkner, M., Dominguez, J., Neshev, D. N., Brener, I., Pertsch, T., and Kivshar, Y. S., "High-efficiency dielectric Huygens' surfaces," Adv. Optical Mater. doi:10.1002/adom.201400584 (2015).

[105] Engheta N. Circuits with Light at Nanoscales: Optical Nanocircuits Inspired by Metamaterials. Science 2007; 317: 1698-1702.

[106] Sun Y, Edwards B, Alù A, Engheta N. Experimental realization of optical lumped nanocircuits at infrared wavelengths. Nature Materials 2012; 11: 208-212.

[107] Zheludev NI, Kivshar YS. From metamaterials to metadevices. Nature Materials 2012; 11: 917-924.

[108] Kaiser T, Flamm D, Schröter S, Duparré M. Complete modal decomposition for optical fibers using CGH-based correlation filters. Optics Express 2009; 17: 9347-9356.

[109] Manek I, Ovchinnikov YB, Grimm R. Generation of a hollow laser beam for atom trapping using an axicon. Optics Communications 1998; 147: 67-70.

[110] Ozaki M, Kato J, Kawata S. Surface-Plasmon Holography with White-Light Illumination. Science 2011; 332: $218-$ 220. 